\title{
Superconducting Current Transfer Devices for Use with a Superconducting LVdc Mesh
}

\author{
B. K. Johnson, Member, IEEE, R. H. Lasseter, Fellow, IEEE, F. L. Alvarado, Fellow, IEEE, \\ and R. Adapa, Senior Member, IEEE
}

\begin{abstract}
This paper describes engineering analysis and simulation results for a device intended to transfer current among parallel superconducting branches in a meshed superconducting dc system. Meshed superconducting de systems offer unique problems of current distribution between parallel paths due to the lack of resistance. Neither resistances nor steady-state impedances can be relied upon to equalize the loading among superconducting branches, or to prevent any specific branch from exceeding its critical current and quenching. The paper describes the design and operation of a superconducting current diverter based on the creation of a normal zone in a specially designed superconducting cable section. The current diverter can operate successfully with a surprisingly small normal state resistance. The main disadvantage of these devices is the loss of energy associated with the resistance of the device when activated. These devices appear promising for both routine line operations and for current steering in meshed superconducting dc systems.

Key Words-Superconductors, current diverter, current steering, mesh connected MTdc system.
\end{abstract}

\section{INTRODUCTION}

$\mathrm{T}$ HE discovery of high temperature superconductivity [1] has sparked a great deal of interest in its application to the power area. One such application, low voltage direct current (LVdc) power transmission [2], [3], may yield a reduction in transmission and distribution costs. Superconducting transmission can connect distant generating stations to load distribution systems, replacing high voltage transmission and subtransmission systems with a low voltage, high current system. A mesh connected system provides parallel transmission paths for added reliability. Fig. 1 shows one pole of a $\pm 7.5 \mathrm{kV}$ bipolar system developed in [3].

A superconducting power transmission system need not operate at high voltage levels to reduce $I^{2} R$ losses. This eliminates the need for step-up transformers, and reduces

Manuscript received February 23, 1994; revised August 12, 1994. This work was part of a project on High Temperature Superconducting dc Networks supported by the National Science Foundation under Grant 8818339 and EPRI Project RP7911-12.

B. K. Johnson is with the University of Idaho.

R. H. Lasseter and F. L. Alvarado are with the University of Wisconsin-Madison.

R. Adapa is with the Electric Power Research Institute, Palo Alto, CA xxxxx.

IEEE Log Number 9406126

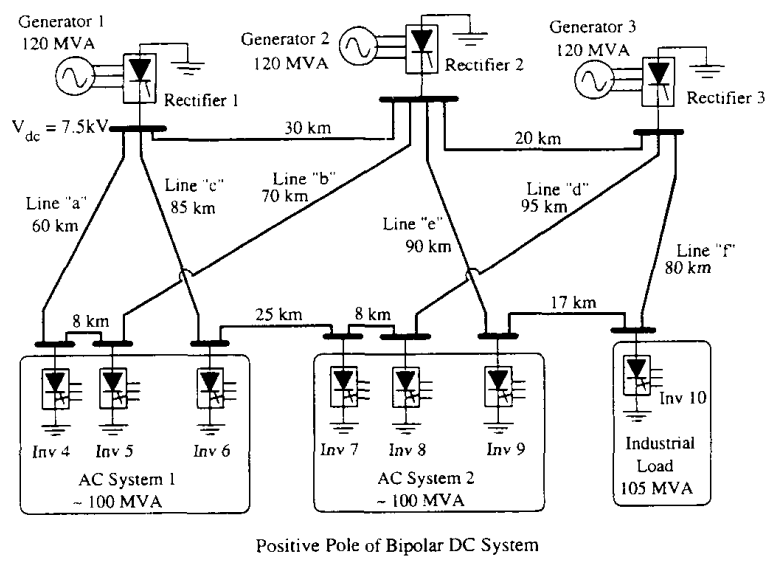

Fig. 1. Ten bus LVdc test system.

high voltage insulation needs. The complete system can operate at optimum generator voltages, resulting in a single voltage level from generation to distribution.

The mesh connection of a dc system presents difficulties with fault detection and protection [4]. The dc faults can be starved through converter action for a point-topoint HVdc transmission system. This is not easily done for a mesh connected system without shutting the entire system down. Direct current circuit breakers capable of operating in an $\mathrm{HVdc}$ transmission system have been developed [5]. However, the addition of a mechanical circuit breaker to a superconducting transmission system may not be the most effective solution. The circuit breaker adds resistance, and connecting it into a line with zero resistance decreases the efficiency of the system. The use of a device that is superconducting when not in use would be more effective. Superconducting fault current limiters have been discussed for use in ac systems [6]-[8], with experimental results given in [6] and [9]. These devices carry current through a superconductor when either the current limit of the superconductor is exceeded, or the increased current through a trigger coil wrapped around the superconductor creates a normal zone in that section of the cable. This results in an increase in either device inductance or device resistance, depending on the design 
of the device. The increase in inductance and resistance is used to limit fault currents.

This paper discusses the use of devices similar to resistive ac fault current limiters in a superconducting LVdc meshed system. Any resistance inserted into a superconducting line causes all of the dc current to be transferred out of the line into the parallel, superconducting paths. Therefore, the device added to the system can have a relatively small resistance, limiting the current transfer rate and resulting overvoltages. Quenching of the superconductor can be controlled by imposing either an external electric or magnetic field [6], [10]. This presents the opportunity to design a device that can be used to transfer just a fraction of the current out of the line, providing the ability to move heavily loaded lines away from their current limits. This paper introduces a superconducting current diverter for use in parallel or mesh connected LVdc transmission systems. The basic current diverter can be used to open a line during routine operation, clear a fault, or perform current steering to balance transmission loading. The basic current diverter concept is introduced, followed by a discussion of applications of the diverter for removing lines, clearing faults, and current steering.

\section{Superconducting Current Diverters}

The ability to remove a line is necessary both for routine maintenance and for clearing faults. This is especially important for a multiterminal dc (MTdc) transmission system. The removal of a line can be done using conventional dc circuit breakers developed for $\mathrm{HVdc}$ transmission applications. Conventional dc breakers may not be suitable for superconducting transmission applications since they add resistance to the system. A device that is superconducting when not in use would not suffer from this problem. This paper illustrates that such a current diverter can be used not only as a breaker, but also for current steering.

A superconducting circuit breaker can be created by forcing a section superconducting material in a controlled zone of the line to go normal, as is done with superconducting current limiters [6], [7], [9] or superconducting power electronic switches [10]. This inserts a resistance into the line, with the resistance increasing over a period ranging from a few microseconds to tens of milliseconds. The time for the resistive rise is partly a design feature, although in this case a very rapid rise is desirable. Faster increases in resistance have been achieved with low temperature superconductors, while high temperature superconductors are limited to slower rates of increase at present [11], [12]. Inserting any resistance into a superconducting line causes all current to transfer into parallel paths in a superconducting de mesh. The energy of the line is dissipated in this resistive section, but is localized to a known section of the line, so that section of the line can be designed with sufficient added refrigeration.

Our analysis and simulation results show this device can operate successfully with a resistance of a few ohms or less. There is no need, nor even a desire, for larger resistances, as they would result in large voltage spikes when the conductor goes normal. The magnitude of the induced overvoltages from the device activation is directly proportional to the resistance inserted into the line. A smaller resistance reduces the overvoltages, but it also increases the current transfer time. The resistance of the normal zone in the superconductor rises rapidly over several tens of microseconds. This is several orders of magnitude faster than the current transfer out of the line, which is based on the $\mathrm{L} / \mathrm{R}$ time constant of the normal zone resistance and the equivalent inductance seen looking at the mesh from this point. The transition appears essentially as a step increase in resistance.

Simply inserting a resistance is not sufficient for clearing a line-to-line fault or a fault to ground. The voltage driving the fault current will now appear across the resistance of the diverter. Adding an LC resonant circuit in parallel with the current diverter can create a current zero. A similar concept is used for conventional $\mathrm{HVdc}$ circuit breakers [5]. The change in voltage across the diverter triggers $\mathrm{LC}$ resonance between the line inductance and the added LC circuit. The resonance forces a transient current zero in the line, allowing a mechanical switch to be opened at the cable termination at the bus. The energy of the LC resonance is absorbed by the ac resistance of the superconducting line and the resistance of the normal zone. Adding a fixed resistance in series with the capacitor damps the oscillations more quickly. Fig. 2 shows a superconducting current diverter with a line $\mathrm{LC}$ resonance task connected in parallel.

\section{Actilating Superconducting Switches}

Activation of the superconducting switch requires creating a normal zone in the superconductor. There are three ways to create a normal zone in a superconductor: excess temperature, excess current, and excess magnetic field. Fig. 3 shows the general configuration for a superconducting current diverter. The auxiliary LC circuit is added only for circuit breaker applications. The diverter has its own refrigeration unit to increase energy dissipation ability. Therefore, the diverter also requires its own pumping station. This device would normally be connected near the end of a transmission line, allowing easier construction of the cooling station and providing an energy supply for the trigger.

Superconducting fault current limiters are generally triggered by one of two methods. The simplest method is simply based on the current through the superconductor surpassing the current limit of the superconductor. This does not provide precise activation and results in a slower change in resistance. A more common method, described in [6] and [9], is based on a magnetic trigger. The line current is run through a magnetic coil connected in series with the diverter itself. This coil is wound around the diverter assembly so that it impresses a magnetic field on the diverter. An increase in current would cause the magnetic field to increase, creating a normal zone in the superconductor. Since this depends on the increase in the 


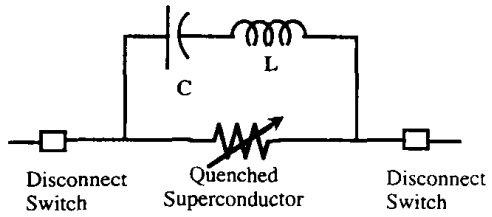

Fig. 2. Superconducting circuit breaker with LC tank to initiate current zero.

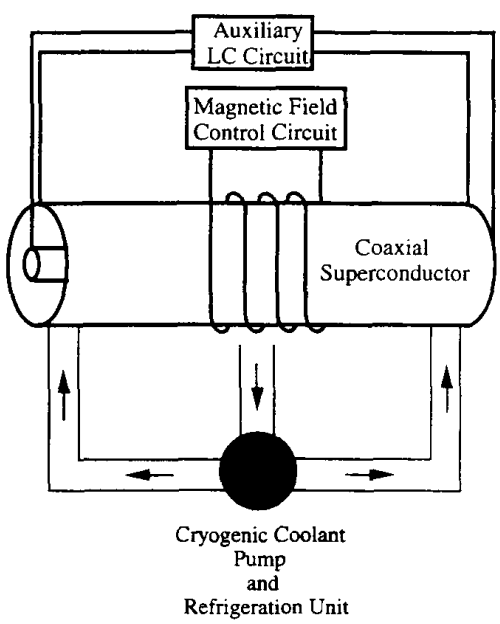

Fig. 3. Superconducting current diverter.

line current, it could again have a slower response in some cases, but has the advantage of providing passive protection for fault conditions.

The superconducting current diverter described here is based on a configuration with a trigger coil wound around the superconductor, as shown in Fig. 3. The trigger coil is fed by a separate power supply, and provides sufficient energy for a rapid increase in resistance in the superconductor. The diverter is triggered on command from either a central controller for system current control or a local relay upon detection of a fault.

\section{Routine Line Opening}

Adding resistance to one line in a mesh connected superconducting dc transmission system causes all of the current in that line to transfer to the parallel transmission paths in the dc system, which are still in a zero resistance superconducting state. The addition of a resistance in a controlled manner can be used to aid in the removal of a line of routine service. The cable can be removed from the transmission system more easily once the line current has gone to zero.

The current diverter provides an effective, controlled means to bring the current to zero. The resistance of a small section of superconductor can be changed from zero to a design level. The value of the resistance depends on the diameter and length of the normal section. The current then transfers out of the line based on the resulting
$\mathrm{L} / \mathrm{R}$ time constant. The resistive voltage drop of the normal zone divides over the length of the line based on the relative inductances. This causes the voltage at the sending end to rise, while the voltage at the receiving end of the line falls. A larger resistance causes a larger overvoltage at the sending end of the line.

\section{Fault Clearing}

The superconducting current diverter discussed in the previous section is adequate for removing the current from a line under normal operating conditions. All of the terminals on the dc system have equal steady-state voltages, so there is no voltage across the resistance of the device to drive steady-state currents, allowing the line to be disconnected from the dc network at the ends of line. However, this is not true if there is a pole-to-pole or a pole-to-ground fault on the dc system. There will be a large voltage across the normal state resistance of the diverter. The added resistance helps limit the rate of rise of the fault current, and helps limit the maximum current, but it does not bring it to zero. A transient current zero can be induced by forming a resonant circuit in parallel with the diverter similar to the process used in the case of a dc circuit breaker, where the arc resistance is replaced by the normal state resistance of the diverter. This induces a transient current zero allowing the mechanical switches to open disconnecting the line from the bus at the ends of the line.

The simple system shown in Fig. 4 can be used to demonstrate circuit breaker action. Fig. 5 shows simulation results for an example with a superconducting breaker opening a faulted line when a resistive fault occurs halfway down the lower line shown in Fig. 4. This example has two parallel transmission lines, each carrying $2400 \mathrm{~A}$ initially, while operating at $10 \mathrm{kV}$. Both lines are fed by a single rectifier and feed a line commutated inverter. A resistive fault occurs in one of the lines, triggering a circuit breaker. The superconducting breaker has a normal resistance of $R_{n}=10 \Omega$, in parallel with a $400 \mu \mathrm{F}$ capacitor. The capacitor resonates with the line inductance which is 2 $\mathrm{mH}$. The lines are based on the cable design presented in [3], and correspond to a cable roughly $60 \mathrm{~km}$ in length. The maximum fault current for the example is $12.5 \mathrm{kA}$. The plot in Fig. 5(a) shows the voltages at each end of the line. Fig. 5(b) shows the line current in the faulted branch. The current increases rapidly due to the fault, and then goes to zero after the breaker acts. Fig. 5(c) shows the receiving end current for the faulted line (lower line), and the current in the parallel line (top line). The current at the receiving end of the faulted line decreases when the fault occurs. A mechanical switch disconnects the line from the inverter bus, preventing current reversal. The parallel line picks up the current that was originally carried in the faulted line. The parallel line current increases initially as it supplies current to the fault by way of the connection to the faulted line at the inverter terminal. The fault current is interrupted by the breaker action, and the current begins to decrease until the trans- 


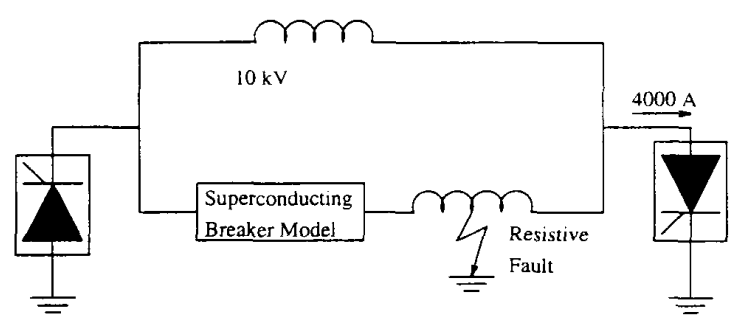

Fig. 4. Sample system for superconducting breaker example.

fer of current from the faulted line begins. Fig. 5(d) shows the current in the capacitor. The capacitor current jumps rapidly due to the change in voltage across the capacitance when the voltage across the now activated diverter changes. This current rings with the line inductance, allowing the line to be disconnected by a mechanical switch at the rectifier terminal. Note this switch wold normally be present for routine line removal, but it will need to be modified to allow the contacts to spread apart rapidly in this case.

The normal resistance and the parallel capacitance can be varied to provide the ability to clear different current levels. This also depends on the inductance in the line. In a practical implementation of this concept, it will probably be necessary to add some inductance in series with the capacitor to be able to clear faults close in to the rectifier bus.

\section{Current SteEring}

The current flows within a meshed dc system are determined by the node voltages and the branch inductances of the mesh. Some form of active control within the branches of the mesh is needed to be able to alter this natural distribution of current. This active control should be lossfree when not in use.

The current in any superconducting line must be below the level where it will quench. An active current steering system could move a threatened line away from its current limit. Another case where current steering is useful is the reinsertion of a line. A line that has been removed from the dc system will carry no current when it is initially switched back into the mesh. This is because the superconducting mesh reaches a steady state with all terminals at equal voltages. Therefore, the newly inserted line will not see a voltage difference across its inductance until a disturbance or operational change on the mesh creates one. The mesh currents will stay in the same state until a change in load current occurs [2]. Repeated removals and reinsertions over time could lead to a case where a few lines operate close to their limits while other paths carry almost no current. This can be avoided by having a current steering system balance the mesh currents when the new line is reinserted.

A global current steering system requires a centralized communication system. However, such a scheme can be relatively slow, so a less expensive communication system can be used. The control scheme communicates set points to the various controllers on the mesh.

\section{Parallel Current Diverters}

The current diverter can be used to transfer part of the current out of the line for current steering. Current steering can be accomplished by activating a single device, and then resetting it before the current has gone to zero. This requires a section of superconductor with sufficient refrigeration to remove the heat rapidly after the controlled normal zone has been created. Such a process should happen quickly enough to limit the line current without decreasing it to zero. However, a resistance of a few ohms is able to transfer all of the current out of a line in $10-15 \mathrm{~ms}$ in simulated cases based on the system developed in [3]. Since the recovery of the superconductor from nonsuperconducting operation is a thermodynamic process, the restoration of a diverter to the superconducting state is likely to take significantly longer than the current transfer.

One possible way to initiate partial current transfer out of a line would be to have two identical superconducting devices connected in parallel. Fig. 6 shows a sample system where a parallel connected current diverter is inserted into one line of a simple system with two parallel lines. Part of the line current is transferred out of the line by activating one of the devices, without activating the other. Quenching one of the devices avoids problems with the slow thermodynamic recovery of the diverters, since there is still a superconducting current path in the line. The current in the normal portion of the line would divide between all of the possible parallel paths in the system, including the other leg of the device. The current redistributes between the different paths to maintain volt-second balance over the mesh. The amount of current transferred depends on the size of the inductance added to the diverter compared to the equivalent inductance seen looking into the remainder of the system from the terminals of the normal device. A larger inductance also increases the amount of energy dissipated when the device is activated. Additional current can be transferred out of the line by activating the second leg of the device after the first leg has resumed a superconducting state.

The values of the normal state resistance and the inductance added to the line by the diverter play an important role in performance. The magnitude of the inductance determines the amount of current transferred out of the line each time the device is pulsed. The magnitude of the resistance has no effect on the amount of current transferred. The amount of current transferred out of the line by pulsing one leg of the device is shown in (1), where $I_{o}$ is the initial current and $L_{e q}$ is the equivalent inductance of the balance of the mesh. The value of the inductance in the second leg relative to $L_{e q}$ is the key to the amount of transfer. The resistance value determines the time constant for the current transfer, and also determines the size of the transient voltage change that will 


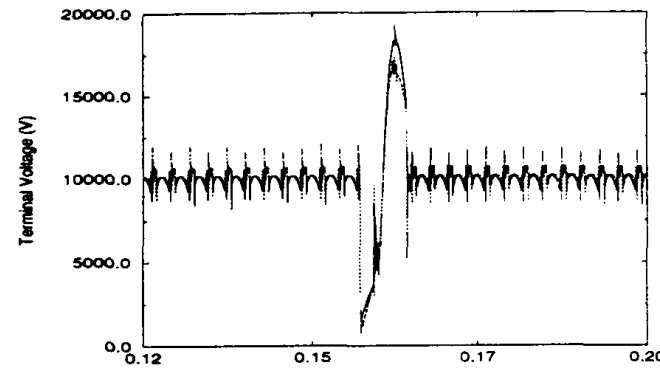

(a)

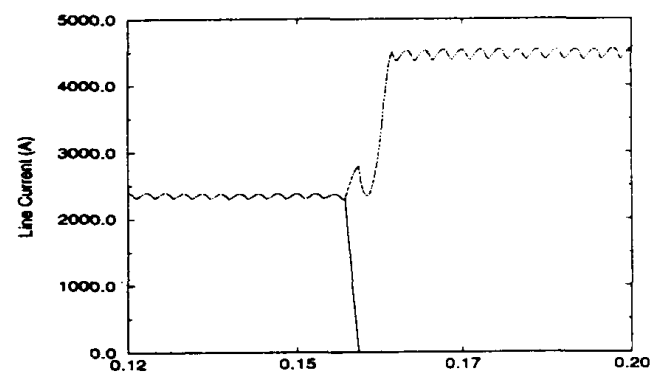

(c)

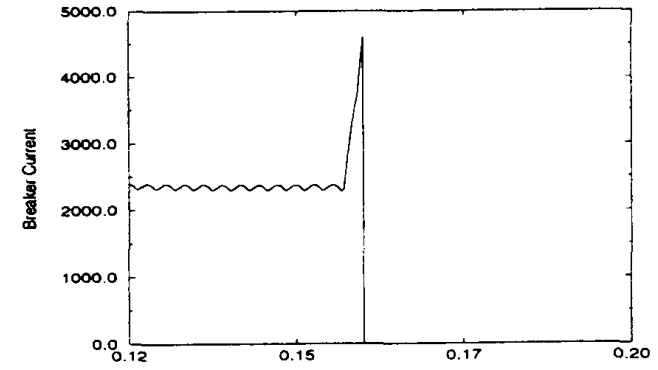

(b)

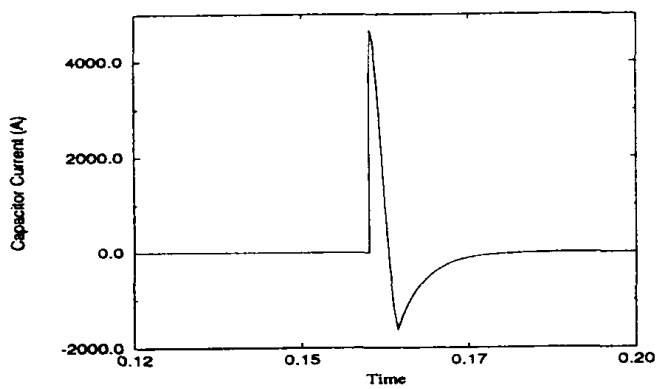

(d)

Fig. 5. Superconducting breaker during dc fault.

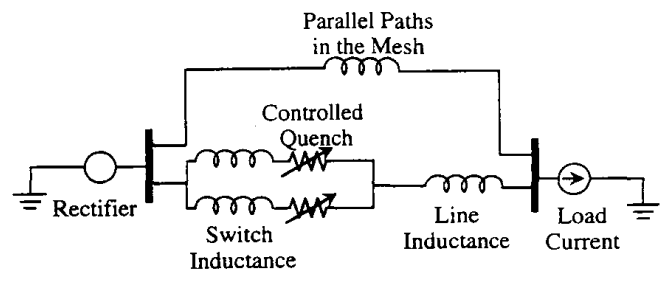

Fig. 6. Superconducting current transfer device with parallel legs.

occur.

$$
\Delta I_{11}=\frac{L_{11} * I_{o}}{L_{12}+L_{e q}+L_{1}} .
$$

The energy dissipation each time one leg of the device is pulsed is also dependent on the inductance added to the steering device. Equation (2) shows this relationship. If the device is pulsed repeatedly, with many small jumps, more energy will be dissipated. Again, $I_{o}$ is the initial current. Larger values of added inductance increase the amount of current transfer, and increase the energy dissipation each time the device is used. Therefore, a design tradeoff needs to be made to choose this value. A smaller inductance allows a more precise transfer if repeated device cycles are used. This causes additional energy to be dissipated each time the device is used. However, the energy declines in each subsequent step since the line current is declining. Therefore, the recovery time of the diverters will decrease as the current decreases.

$$
\begin{aligned}
E_{\text {diss }} & =\frac{1}{2} L_{f i c} I_{o}^{2} \\
L_{f i c} & =L_{11}+L_{12} \|\left(L_{1}+L_{e q}\right) .
\end{aligned}
$$

\section{Simulation Results}

Superconducting current steering devices were added to the six main lines in the $\pm 7.5 \mathrm{kV}$ system shown in Fig. 1. Each of these lines typically carries a current of around 2-3 kA. These are the lines that connect the rectifier terminals to the inverter terminals. The devices were sized so that pulsing one leg on a device will transfer a maximum of $2 \%$ of the current out of the line; this is assuming that the other leg carries no current. Each line will require an added inductance in the range of $0.05-0.1 \mathrm{mH}$ to transfer the desired current. Each device has a normal state resistance of $R_{n}=1 \Omega$. The diverters are controlled to deactivate whenever the current is within $\pm 20 \%$ of the set point. A period of $0.4 \mathrm{~s}$ was allowed for the superconductor to recover and resume a superconducting state. This time period was chosen a bit short to decrease simulation time. The legs of each diverter are alternately pulsed until the line current reaches the desired level.

The sample system shown in Fig. 1 has the six long transmission lines set up in three groups of two lines. Two lines supply current to inverters 4 and 5 (lines $a$ and $b$ ); lines $c$ and $d$ supply current to inverters 6,7 , and 8; and lines $e$ and $f$ supply current to inverters 9 and 10. This pairing localizes the effects of transients to smaller parts of the system. Also note the cross connection of the load systems. The pairing results in simpler constraints for the set points of the current steering devices. The set points 

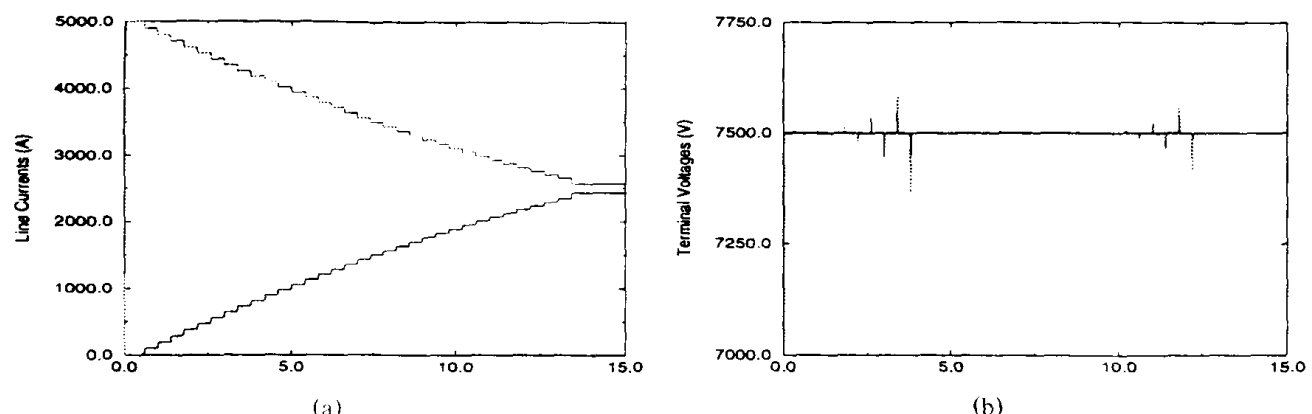

(b)

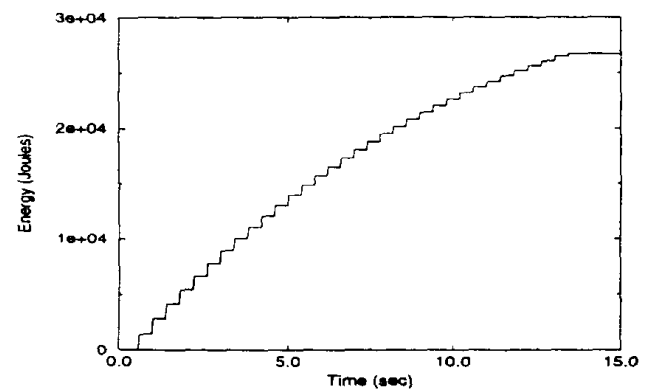

(c)

Fig. 7. Current steering to restore current in line $a$ after reinsertion. (a) Currents in line $b$ (top), and $a$ (bottom). (b) de voltage at rectifier 1. (c) Energy dissipated in line between rectifier 2 and inverter 5 .

of the current steering devices must always be compatible with the inverter current orders.

Fig. 7 shows a case where one of the two lines feeding inverters 4 and 5 is reclosed. Fig. 7(a) shows the line currents. One line carries the full load, and the other carries no current. The other line is reinserted after 200 $\mathrm{ms}$, but does not carry current until the current steering device is activated after $500 \mathrm{~ms}$. The current gradually moves toward the desired point, and then toward a steady state. The slow response is due to the recovery time set for the superconductors. Fig. 7(b) shows the dc voltage at the sending end of the lines. The voltage experiences small spikes whenever the devices are activated. The inverter currents are largely unaffected by the operation of the current diverters, since the voltage spikes are too small to cause significant current changes. Fig. 7(c) shows the energy dissipation in the resistances of the normal zones. The energy dissipation increases in a stairstep fashion every time one of the legs is activated.

The total energy transferred through these two lines in this time period was around $2000 \mathrm{MJ}$. The diverter was pulsed 33 times $(17$ times for one leg, and 16 for the other) to bring the currents to the desired levels. The current diverter dissipated $26.6 \mathrm{~kJ}$ during this time, resulting in a very small percentage energy loss. The initial pulse begins with the full line current of $4999 \mathrm{~A}$. The device transfers about $93 \mathrm{~A}$, or little less than $2 \%$ of the current out of the leg. It dissipates about $1.4 \mathrm{~kJ}$ in the first step. Although energy losses are small, efficiency considerations limit current steering to necessary situations.

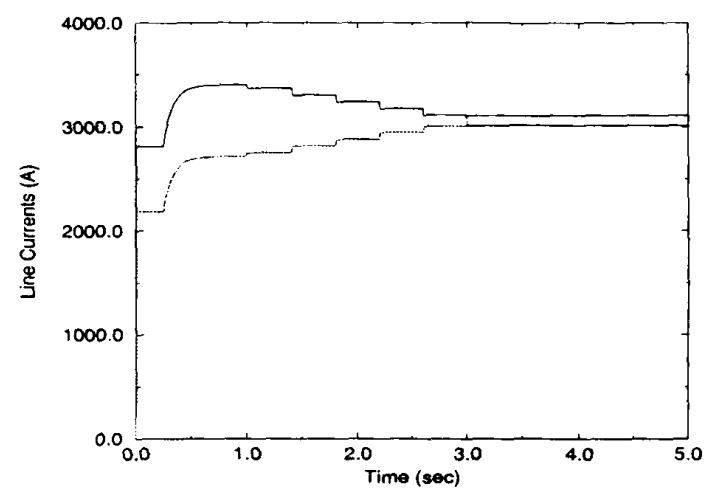

Fig. 8 . Using current steering to balance line currents.

Fig. 8 shows a case where the current drawn by inverter 4 is increased. The increase in current divides unevenly between lines $a$ and $b$ coming from the rectifiers (as shown in Fig. 1) due to differences in path inductances. The current steering devices are activated to balance the currents between the two lines. The figure shows the result of the current diverter action on the line currents in lines $a$ and $b$. Again, the diverters allow $0.4 \mathrm{~s}$ for the superconductors to recover from creation of the normal zone.

\section{Conclusion}

This paper has introduced a superconducting current diverter based on the controlled creation of a normal 
zone in a short section of a superconducting dc cable. With suitable controls and additional parallel components, the current diverter can be used for routine line removal, clearing faults, and current steering. The addition of a resistance to a superconducting the line transfers current out of that line to parallel superconducting paths, allowing simple removal of the line.

Current diverters have some drawbacks. Losses are incurred whenever the device is activated due to resistive heating of the current diverter, which needs a higher capacity cooling system. The current diverters also require a pulse of energy to force a normal zone in a controlled section the superconducting cable. The energy losses incurred through the use of these devices are a very small fraction of the total energy transfer in the dc system. Nevertheless, actual activation of the device should occur only when required to maintain currents within acceptable ranges. Current diverters can be implemented with either low or high temperature superconductors.

\section{REFERENCES}

[1] J. G. Bednorz and K. A. Müller, Possible High $T_{c}$ Superconductivity in the Ba-La-Cu-O System. Zeits: Physik, vol. B64, 1986, p. 189.

[2] B. K. Johnson, R. H. Lasseter, and R. Adapa, "Power control applications on a superconducting LVDC mesh," IEEE Trans. Power Delivery, vol. 6, pp. 1282-1288, July 1991.

[3] R. H. Lasseter, F. L. Alvarado, D. M. Divan, B. K. Johnson, H. Singh, and M. C. Chandorkar, "Superconducting LVDC networks," EPRI TR-103636, Electric Power Research Institute, Palo Alto, CA, Apr. 1994

[4] K. W. Kanngiesser, J. P. Bowles, ̊ Ekström, J. Reeve, and E Rumpf, "HVDC multiterminal systems," CIGRE, vol. 14-08 (ELECTRA), Aug. 1974.

[5] "500 kV HVDC air blast circuit breaker," BBC, Brown Boveri, and Cie., Final Rep., Aug. 1986, EPRI Rep. EL-4520, RP-1507-03.

[6] V. D. Pham, Y. Lamoud, T. Verhaege, A. Fevrier, M. Collet, and M. Bekhaled, "Towards the superconducting fault current limiter," IEEE Trans. Power Delivery, vol. 6, pp. 801-808, Apr. 1991.

[7] T. Ishigohka and N. Sasaki, "Fundamental test of new superconducting fault current limiter," IEEE Trans. Magn., vol. 27, pp. 2341-2344, Mar. 1991

[8] N. M. Wilson, Superconducting Magnets. New York: Oxford University Press, 1983.

[9] T. Hara, T. Okuma, T. Yamamoto, D. Ito, K. Tasaki, and K. Tsurunaga, "Development of a new $6.6 \mathrm{kV} / 1500 \mathrm{~A}$ class superconducting fault current limiter for electric power systems," IEEE Trans. Power Delivery, vol. 8, pp. 182-192, Jan. 1993

[10] S. J. Dale, S. M. Wolf, and T. R. Schneider, "Energy applications of high temperature superconductivity. Vol. 2: Detailed assessment," EPRI, ER-6682, Nov. 1990.
[11] T. Ikegami, Y. Yamagata, and K. Ebihara, "Application of high-Tc superconductor to current limiting devices," IEEE Trans. Appl. Superconduct., vol. 3, pp. 566-569, Mar. 1993.

[12] M. Lindmayer and M. Schubert, "Resistive fault current limiters with HTSC-Measurement and simulation," IEEE Trans. Appl. Superconduct., vol. 3, pp. 884-888, Mar. 1993.

[13] "Energy applications of high-temperature superconductors: A progress report," EPRI, TR-101635, July 1992.

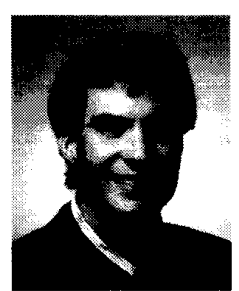

Brian K. Johnson (S'86) received the Ph.D. degree in Electrical Engineering in 1992 from the University of Wisconsin-Madison.

$\mathrm{He}$ is current an Assistant Professor in the Department of Electrical Engineering at the University of Idaho. His interests include $\mathrm{HVdc}$ transmission, power systems, and power electronics.

Robert H. Lasseter (F'92) received the Ph.D. degree in physics at the University of Pennsylvania, Philadelphia, in 1971.

He was a Consultant Engineer at General Electric Company until he joined the University of Wisconsin-Madison in 1980. His main interests are the application of power electronics to utility systems and simulation methods.

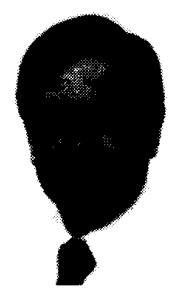

Fernando L. Alvarado (F'93) obtained a Ph.D degree from the University of Michigan.

He is currently a Professor at the University of Wisconsin in Madison in the Department of Electrical and Computer Engineering. His main interests are in computer applications to power systems and large scale problems.

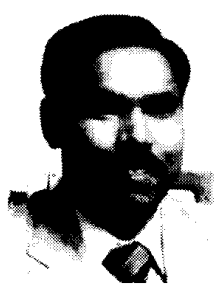

Rambabu Adapa received his $\mathrm{Ph} . \mathrm{D}$. degree in electrical engineering from the University of Waterloo, Ontario, Canada in 1986.

He joined the Power System Planning and Operation program at the Electric Power Research Institute (EPRI), Palo Alto, California in June 1989. Prior to joining EPRI, he was Staff Engineer in the Systems Engineering Department of McGraw Edison Power Systems, Franksville, Wisconsin. 